\title{
White Blood Cell Decreased, CTCAE
}

National Cancer Institute

\section{Source}

National Cancer Institute. White Blood Cell Decreased, CT CAE. NCI Thesaurus. Code C143948.

A finding based on laboratory test results that indicate an decrease in number of white blood cells in a blood specimen. 